\title{
Fluconazole treatment of intrauterine Candida albicans infection in fetal sheep
}

\author{
Gunlawadee Maneenil ${ }^{1,2}$, Matthew S. Payne ${ }^{3}$, Paranthaman Senthamarai Kannan', Suhas G. Kallapur ${ }^{1,3}$, \\ Boris W. Kramer ${ }^{3,4}$, John P. Newnham ${ }^{3}$, Yuichiro Miura ${ }^{3}$, Alan H. Jobe ${ }^{1,3}$ and Matthew W. Kemp ${ }^{3}$
}

BACKGROUND: Intrauterine Candida albicans infection causes severe fetal inflammatory responses and fetal injury in an ovine model. We hypothesized that intra-amniotic antifungal therapy with fluconazole would decrease the adverse fetal effects of intra-amniotic C. albicans in sheep.

METHODS: Sheep received an intra-amniotic injection of $10^{7}$ colony-forming units C. albicans. After $2 \mathrm{~d}$, animals were then randomized to: (i) intra-amniotic and fetal intraperitoneal saline with delivery after $24 \mathrm{~h}$ ( $3 \mathrm{~d}$ C. albicans group); (ii) intra-amniotic and fetal intraperitoneal injections of fluconazole with delivery after either 24h (3 d C. albicans plus $1 \mathrm{~d}$ fluconazole group) or $72 \mathrm{~h}$ ( $5 \mathrm{~d}$ C. albicans plus $3 \mathrm{~d}$ fluconazole group). Controls received intra-amniotic injections of saline followed by intraamniotic and fetal intraperitoneal fluconazole injections.

RESULTS: Intra-amniotic C. albicans caused severe fetal inflammatory responses characterized by decreases in lymphocytes and platelets, an increase in posterior mediastinal lymph node weight and proinflammatory mRNA responses in the fetal lung, liver, and spleen. Fluconazole treatment temporarily decreased the pulmonary and chorioamnion inflammatory responses.

CONCLUSION: The severe fetal inflammatory responses caused by intra-amniotic C. albicans infection were transiently decreased with fluconazole. A timely fetal delivery of antimicrobial agents may prevent fetal injury associated with intrauterine infection.

$\mathbf{P}$ reventing preterm birth (delivery before $37 \mathrm{wk}$ completed gestation) is a major challenge in perinatal medicine with the potential to prevent $\sim 1$ million perinatal deaths each year. Although the causes of preterm birth are multifactorial, intrauterine infection is frequently implicated (1). Many intrauterine infections associated with preterm labor are indolent, not clinically apparent and polymicrobial in nature $(2,3)$. Although the most common microorganisms associated with preterm delivery that are identified by culture and molecular-based analyses are bacteria such as the Fusobacterium, Ureaplasma,
Mycoplasma, Streptococcus, Bacteroides, and Prevotella spp., up to $40 \%$ of pregnant woman may also have vaginal colonization with the yeast, Candida spp. $(2,4)$.

The detection of Candida spp. is twofold higher than for nonpregnant women (5), and Candida spp. have been isolated from the amniotic fluid of women with spontaneous preterm birth (6-8). Although congenital candidiasis is an uncommon clinical finding, recent molecular data suggest that $C$. albicans may colonize the amniotic cavity more frequently than initially indicated by culture-based analyses (7). Indwelling contraceptives and cervical cerclage are also associated with funisitis and chorioamnionitis caused by Candida spp. (2,9). Intra-amniotic Candida spp. infection can cause fetal death or fetal candidiasis with impaired neurodevelopmental outcomes $(10,11)$. Again, although vaginal Candida spp. colonization is not frequently associated with increased risk of preterm delivery or low birth weight (12), there is some evidence that eradication of Candida spp. in pregnancy may reduce the risk of late miscarriage and preterm birth. A large retrospective cohort study demonstrated that the use of clotrimazole was associated with a significant reduction in preterm births and an increase in mean gestational age at birth (13). A recent study also reported that nearly $20 \%$ of women had asymptomatic vaginal candidiasis and there was a tendency toward reduction in spontaneous preterm birth among women who were treated with clotrimazole (14).

We previously described models of the fetal inflammatory response syndrome in sheep using the proinflammatory mediators Escherichia coli lipopolysaccharides (15), interleukin (IL)-1 (16), and live Ureaplasma parvum (17). In contrast to the low-grade fetal inflammation caused by these agonists, we recently reported that intra-amniotic $C$. albicans caused a severe, progressive fetal inflammation within $2 \mathrm{~d}$, with the severity of skin, lung inflammatory, and systemic responses threatening fetal viability (18).

Recent studies have described cases of intra-amniotic C. albicans infection that were successfully treated with

The first two authors contributed equally to this work.

'Division of Pulmonary Biology, Cincinnati Children's Hospital Medical Center, University of Cincinnati School of Medicine, Cincinnati, Ohio; ${ }^{2}$ Department of Pediatrics, Faculty of Medicine, Prince of Songkla University, Songkhla, Thailand; ${ }^{3}$ School of Women's and Infants' Health, The University of Western Australia, Perth, Australia; ${ }^{4}$ Department of Pediatrics, School of Oncology and Development Biology, Maastricht University Medical Center, Maastricht, The Netherlands. Correspondence: Matthew W. Kemp (matthew.kemp@uwa.edu.au) 
intra-amniotic fluconazole (19). Fluconazole is a trizaole antifungal agent that is commonly used to treat vaginal candidiasis. Although there are some concerns regarding the use of fluconazole in pregnancy, its efficacy against Candida spp., long half-life and gastrointestinal uptake make it a potentially useful agent to treat intrauterine infections caused by C. albicans (20-22). Therefore, we hypothesized that timely antifungal treatment with fluconazole would decrease the adverse fetal effects of intra-amniotic C. albicans in a pregnant sheep model.

\section{RESULTS}

\section{Outcomes at Delivery and Cells in Cord Blood}

The fetal mortality rate was $14 \%$ (6/40 animals) (Table 1). One saline-treated control fetus died prior to delivery. $25 \%$ of the 3 $\mathrm{d}$ C. albicans-plus $1 \mathrm{~d}$ fluconazole and $37 \%$ of the $5 \mathrm{~d}$ C. albicans plus $3 \mathrm{~d}$ fluconazole-exposed animals died. We did not include a 5 d C. albicans only group as we anticipated 100\% mortality based on the significant degree of $C$. albicans infectionassociated pathology identified in our earlier work with this model system (18). There were no differences in birth weights between saline-only and fluconazole-only control treatment animals, and these were combined as a single control group. Lymphocyte counts for the $3 \mathrm{~d} C$. albicans group significantly decreased relative to control $(P<0.05)$. In contrast, there were no significant changes in neutrophil and monocyte counts between groups. Platelets significantly decreased in the $3 \mathrm{~d} C$. albicans group and $3 \mathrm{~d} C$. albicans plus $1 \mathrm{~d}$ fluconazole group relative to control $(P<0.05)$. Thus, intra-amniotic $C$. albicans infection caused a systemic fetal inflammatory response as indicated by lymphopenia and thrombocytopenia in fetal blood counts.

\section{Detection of C. albicans}

All animals in the $3 \mathrm{~d} C$. albicans group had positive cultures for C. albicans in amniotic fluid (Table 1). All of C. albicansexposed animals that were treated with fluconazole also had positive cultures for $C$. albicans in the amniotic fluid. The majority of the C. albicans-exposed fetuses also had positive blood cultures, irrespective of fluconazole treatment 1 or $3 \mathrm{~d}$ prior to delivery. qPCR analysis demonstrated significantly increased C. albicans RNA in the fetal lung in all groups relative to control $(P<0.05)$. The amount of $C$. albicans RNA trended to be less for the $3 \mathrm{~d} C$. albicans plus $1 \mathrm{~d}$ fluconazole group than for $3 \mathrm{~d} C$. albicans group $(P=0.06)$. No $C$. albicans RNA was detected in the fetal liver in any of the groups (Table 1 ).

\section{Inflammation in Fetal Lungs}

Pulmonary inflammation was evaluated by measuring mRNA expression for proinflammatory cytokines (Figure 1). mRNA for IL-1 $\beta$, IL- 8 , MCP-1, and TNF- $\alpha$ in the fetal lung greatly increased in all C. albicans-exposed groups relative to control. mRNA expression for all cytokines significantly increased in the $3 \mathrm{~d} C$. albicans group and $5 \mathrm{~d} C$. albicans plus $3 \mathrm{~d}$ fluconazole group $(P<0.05)$, except for IL-1 $\alpha$, which only significantly increased in the $5 \mathrm{~d} C$. albicans plus $3 \mathrm{~d}$ fluconazole group $(P<0.05)$. Interestingly, mRNA expression for IL-6 and MCP-1 in the lung significantly decreased in the $3 \mathrm{~d}$ C. albicans plus $1 \mathrm{~d}$ fluconazole group relative to $3 \mathrm{~d} C$. albicans group ( $P=0.002$ and $P=0.008$, respectively).

To better demonstrate the cellular inflammatory response and activation in the lung, inflammatory cell counts were performed for cells expressing CD3 (T-cell), PU.1 (a maturation marker for myeloid and lymphoid cells, found in high levels in

Table 1. Birth weight, and total and differential white blood cell counts

\begin{tabular}{|c|c|c|c|c|}
\hline & Control & $3 \mathrm{~d}$ C. albicans & $\begin{array}{l}3 \mathrm{~d} \text { C. albicans }+ \\
1 \mathrm{~d} \text { fluconazole }\end{array}$ & $\begin{array}{l}5 \mathrm{~d} \text { C. albicans + } \\
3 \mathrm{~d} \text { fluconazole }\end{array}$ \\
\hline Fetal death & 1 & 0 & 2 & 3 \\
\hline Alive at birth & 15 & 7 & 6 & 5 \\
\hline Birth weight, kg & $2.8 \pm 0.3$ & $2.5 \pm 0.2$ & $2.8 \pm 0.4$ & $2.6 \pm 0.3$ \\
\hline Arterial blood pH & $7.2 \pm 0.07$ & $7.2 \pm 0.09$ & $7.3 \pm 0.07$ & $7.05 \pm 0.07^{* *}$ \\
\hline $\mathrm{PaCO}_{2}$ & $70.7 \pm 9.7$ & $77.0 \pm 12.3$ & $65.2 \pm 11.0$ & $96.6 \pm 8.2^{* *}$ \\
\hline $\mathrm{PaO}_{2}$ & $5.6 \pm 1.7$ & $5.8 \pm 3.9$ & $6.5 \pm 2.1$ & $5.5 \pm 1.3$ \\
\hline \multicolumn{5}{|l|}{ Blood counts } \\
\hline White blood cells, $10^{9} / \mathrm{l}$ & $2.7 \pm 1.1$ & $1.4 \pm 0.8$ & $2.2 \pm 0.9$ & $2.3 \pm 1.2$ \\
\hline Neutrophil, $10^{9} / l$ & $0.2 \pm 0.1$ & $0.1 \pm 0.3$ & $0.2 \pm 0.3$ & $0.4 \pm 0.4$ \\
\hline Lymphocyte, $10^{9} /$ I & $1.7 \pm 0.8$ & $0.7 \pm 0.4^{*}$ & $1.2 \pm 0.5$ & $1.2 \pm 0.9$ \\
\hline Monocyte, $10^{9} / /$ & $0.1 \pm 0.06$ & $0.1 \pm 0.1$ & $0.08 \pm 0.06$ & $0.2 \pm 0.2$ \\
\hline Platelet, $10^{9} / \mathrm{I}$ & $606 \pm 157$ & $297 \pm 108^{*}$ & $387 \pm 84^{*}$ & $408 \pm 105$ \\
\hline \multicolumn{5}{|l|}{$\%$ Culture of C. albicans } \\
\hline Amniotic fluid & 0 & $100 \%$ & $100 \%$ & $100 \%$ \\
\hline Blood & 0 & $71 \%$ & $83 \%$ & $60 \%$ \\
\hline C. albcans RNA, $\mu \mathrm{g} R \mathrm{RN} / \mu \mathrm{l}$, lung & 0 & $17.4 \pm 35.5$ & $1.6 \pm 1.3$ & $10.4 \pm 11.1$ \\
\hline
\end{tabular}

Values are mean \pm SD.

${ }^{*} P<0.05$ vs. control. ${ }^{* *} P<0.05$ vs. all groups. 
mature macrophages) and myeloperoxidase (MPO) (a marker for activated neutrophils and monocytes). CD3-positive cell counts significantly increased in the $3 \mathrm{~d} C$. albicans group and $5 \mathrm{~d} C$. albicans plus $3 \mathrm{~d}$ fluconazole group relative to control $(P<0.05)$ (Figure 2a) and counts for MPO-positive cells significantly increased in the $3 \mathrm{~d} C$. albicans group relative to control $(P<0.05)$ (Figure $2 \mathbf{b})$. PU.1-positive cell counts also significantly increased in the $3 \mathrm{~d} C$. albicans group $(P<0.05)$ (Figure 2c). Consistent with the mRNA data, CD3- and PU.1-positive cell counts significantly decreased in the $3 \mathrm{~d}$ C. albicans plus $1 \mathrm{~d}$ fluconazole group compared with the 3 d $C$. albicans group $(P=0.004$ and $P=0.008$, respectively). Immunohistochemical analysis of lung sections also demonstrated that there was a significant decrease in PU.1-positive cells in the $3 \mathrm{~d}$ C. albicans plus $1 \mathrm{~d}$ fluconazole group compared with the $3 \mathrm{~d} C$. albicans group (Figure 2d).

Lung gas volume measured at $5,10,15,20$, and $40 \mathrm{cmH}_{2} \mathrm{O}$ significantly decreased in the $5 \mathrm{~d}$ C. albicans plus $3 \mathrm{~d}$ fluconazole group relative to control. In the $3 \mathrm{~d} C$. albicans group, the lung gas volume also significantly decreased at 5, 10, 20, and 40 $\mathrm{cmH}_{2} \mathrm{O}$ relative to control. The lung gas volume significantly increased in the $3 \mathrm{~d} C$. albicans plus $1 \mathrm{~d}$ fluconazole group at 10,20 , and $40 \mathrm{cmH}_{2} \mathrm{O}$ compared with the $3 \mathrm{~d}$ C. albicans group. (Figure 3a) The increase in SP-A, SP-B, and SP-D mRNA levels indicates a lung maturation signal (Figures $3 \mathbf{b}-\mathbf{d}$ ). SP-A mRNA levels were significantly increased in the $3 \mathrm{~d}$ C. albicans group and $5 \mathrm{~d} C$. albicans plus $3 \mathrm{~d}$ fluconazole group relative to control $(P<0.05)$. SP-B mRNA levels were significantly increased only in the $3 \mathrm{~d} C$. albicans group and SP-D mRNA levels trended to increase in all groups relative to control.

\section{Inflammation in Other Organs}

Inflammation of the chorioamnion was assessed by measuring expression of proinflammatory cytokines (Figure $4 a-c$ ). mRNA expression of IL- 6 significantly increased in the 5 d C. albicans plus $3 \mathrm{~d}$ fluconazole group relative to control $(P<0.05)$. In the $3 \mathrm{~d} C$. albicans plus $1 \mathrm{~d}$ fluconazole group, mRNA expression for IL-1 $\beta$, IL-6, and MCP-1 significantly decreased relative to the $3 \mathrm{~d} C$. albicans group $(P=0.03$, $P=0.001$, and $P=0.03$, respectively). No changes in IL-1 $\alpha$, IL-8, and TNF- $\alpha$ mRNA expression were detected (data not shown). Histology evaluated with hematoxylin and eosin staining demonstrated minimal inflammatory cells in the chorioamnion (data not shown). The membranes were clear except for large white plaques (Figure 4d).

We evaluated the weight of the posterior of mediastinal lymph node as this node receives lymphatic flow from the lung and gastrointestinal tract (23). The posterior of mediastinal lymph node to body weight ratio significantly increased in the $3 \mathrm{~d}$ C. albicans group and $5 \mathrm{~d}$ C. albicans plus $3 \mathrm{~d}$ fluconazole group relative to control $(P<0.05)$ (Figure 5a). The counts for MPO-positive cells in posterior of mediastinal lymph node a

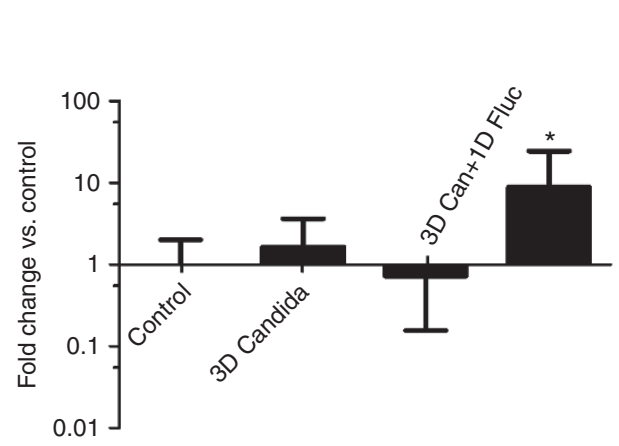

d

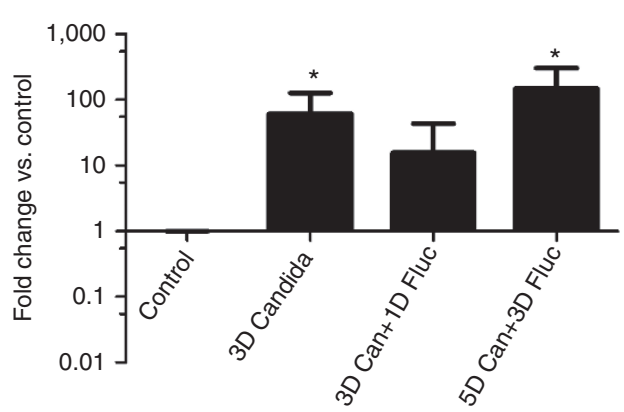

b

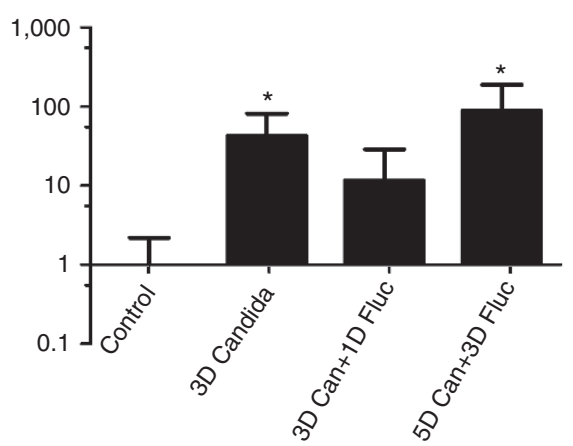

e

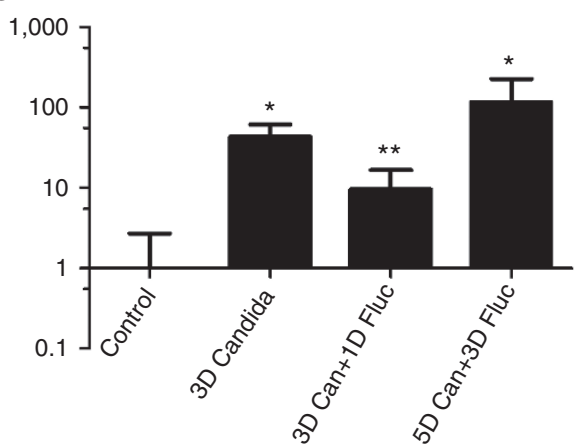

C

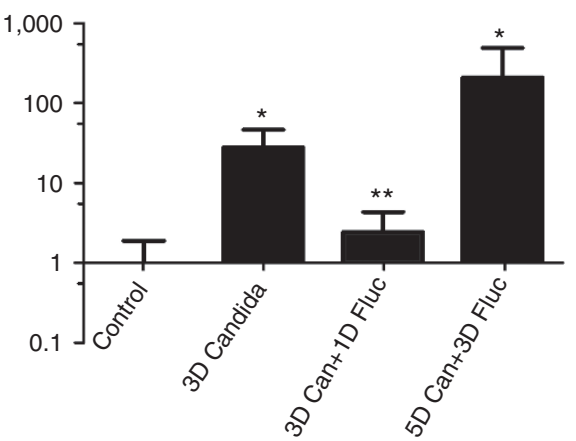

f

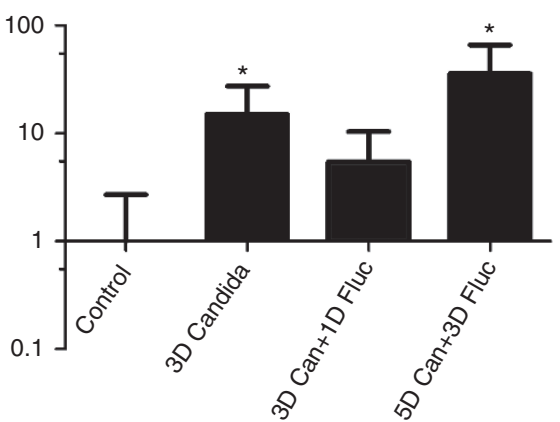

Figure 1. Lung cytokine expression following intra-amniotic C. albicans infection and fluconazole treatment. (a-f) Quantification of $m R N A s$ for IL-1 $\alpha$, IL-1 $\beta$, IL-6, IL-8, MCP-1, and TNF- $\alpha$, respectively, was performed using real-time PCR with ovine-specific primers. Levels for each group were expressed as fold increase relative to control. mRNA expression for IL-1 $\beta$, IL-6, IL-8, MCP-1, and TNF- $\alpha$ was significantly increased in the $3 \mathrm{~d} C$. albicans group and $5 \mathrm{~d}$ C. albicans plus $3 \mathrm{~d}$ fluconazole group relative to control. mRNA expression for IL-6 and MCP-1 was significantly decreased for the $3 \mathrm{~d} C$. albicans plus $1 \mathrm{~d}$ fluconazole group relative to the $3 \mathrm{~d}$ C. albicans group ( $* P<0.05$ vs. control, $* * P<0.05$ vs. the $3 \mathrm{~d} C$. albicans group). 
a

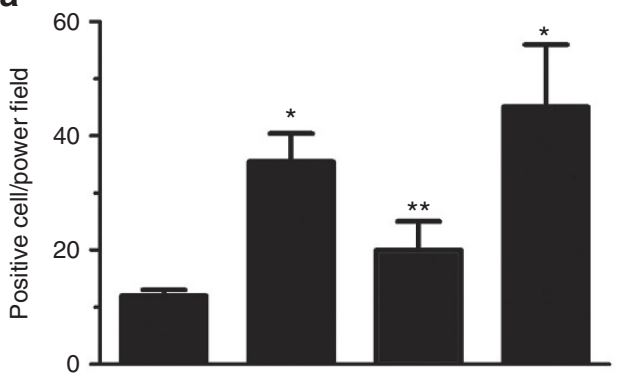

C

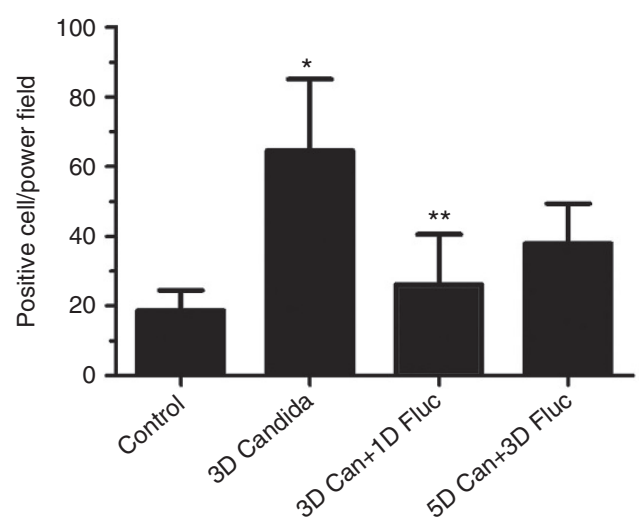

b

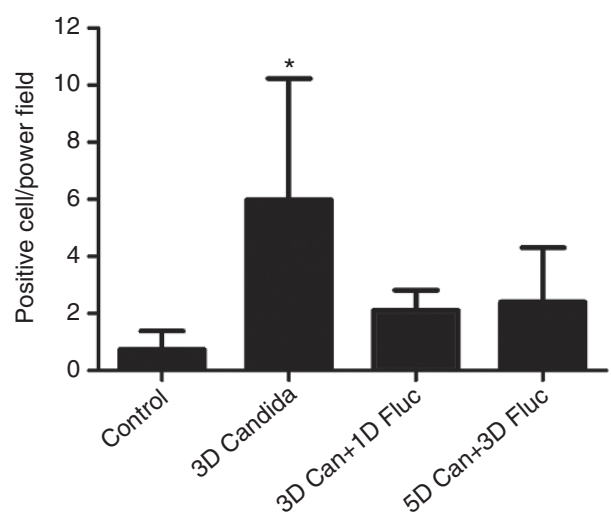

d
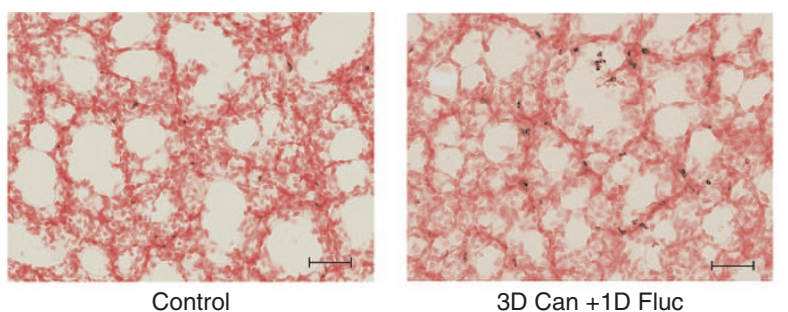

3D Can +1D Fluc

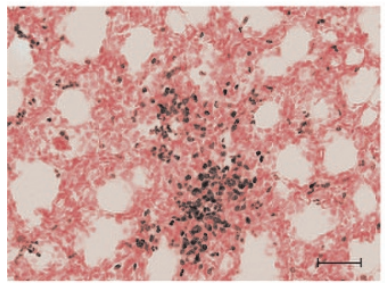

3D Candida

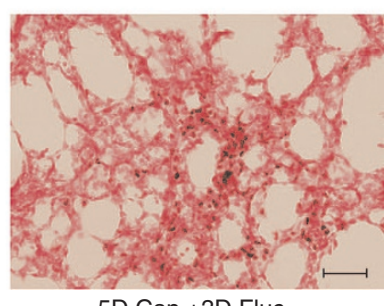

5D Can +3D Fluc

Figure 2. CD3, myeloperoxidase (MPO), and PU.1 expression in the lung following intrauterine C. albicans infection and fluconazole treatment. (a) CD3-positive cell counts significantly increased in the $3 \mathrm{~d}$ C. albicans group and $5 \mathrm{~d}$ C. albicans plus $3 \mathrm{~d}$ fluconazole group relative to control. (b) Counts for MPO-positive cells significantly increased in the $3 \mathrm{~d}$ C. albicans group relative to control. (c) PU.1-positive cell counts significantly increased in the 3 $\mathrm{d}$ C. albicans group relative to control and significantly decreased in the $3 \mathrm{~d}$ C. albicans plus $1 \mathrm{~d}$ fluconazole group relative to the $3 \mathrm{~d}$ C. albicans group. (d) Representative photomicrographs are shown for PU.1 immunostaining. All images $40 \times$ magnification and the scale bar showed $50 \mathrm{~mm}$. The immunostained inflammatory cells (dark brown) were significantly increased in the $3 \mathrm{~d}$ C. albicans group $* P<0.05$ vs. control, $* * P<0.05$ vs. the $3 \mathrm{~d} C$. albicans group).

tended to increase in the $3 \mathrm{~d}$ C. albicans group but decreased in both 3 and $5 \mathrm{~d}$ C. albicans and fluconazole-exposed groups relative to control (data not shown). Hepatic inflammation was assessed by measuring mRNA expression for the acute phase response proteins, SAA3 and C-reactive protein (CRP). SAA3 and CRP mRNA expression was significantly increased in all groups relative to control $(P<0.05)$ (Figure $5 \mathbf{b}, \mathbf{c}$ ). In the fetal spleen, mRNA for IL-6 significantly increased in the $3 \mathrm{~d} C$. albicans group and $5 \mathrm{~d}$ C. albicans plus $3 \mathrm{~d}$ fluconazole group relative to control $(P<0.05)$ (Figure 5d).

\section{DISCUSSION}

Systemic fungal and yeast infections are a significant problem for infants requiring intensive care, and the major yeast pathogen in preterm infants is C. albicans $(10,24)$. In healthy pregnant women, Candida spp. are common vaginal organisms that rarely infect the normal fetus. However, Candida spp. can cause ascending infection in association with preterm labor or preterm rupture of membranes (25). Candida spp. have been cultured from amniotic fluid of women with spontaneous preterm birth $(7,26)$. In humans and animal models, there is limited information about the inflammatory responses of the fetus to C. albicans. We previously described the multiorgan fetal inflammatory responses to intra-amniotic C. albicans in an ovine model of pregnancy (19). In this current study, we replicate this inflammatory response and demonstrate that a single dose of the fungal-static drug fluconazole initially suppresses the fetal infection and associated fetal inflammatory response but is unlikely to be effective in a single-dose regimen for the treatment of intra-amniotic C. albicans infection.

The characteristics of the fetal inflammatory response caused by $C$. albicans colonization of the amniotic cavity should be interpreted within the context of other sheep models of chorioamnionitis and fetal inflammatory response syndrome. An intra-amniotic injection of lipopolysaccharides causes acute chorioamnionitis, striking lung inflammation, lung maturation, and a mild systemic inflammation in multiple organs including the gut, liver, skin, and the immune system (spleen, thymus, lymph node) $(15,27)$. In contrast, $U$. parvum colonizes the amniotic fluid, fetal membranes, and lung but with 
a

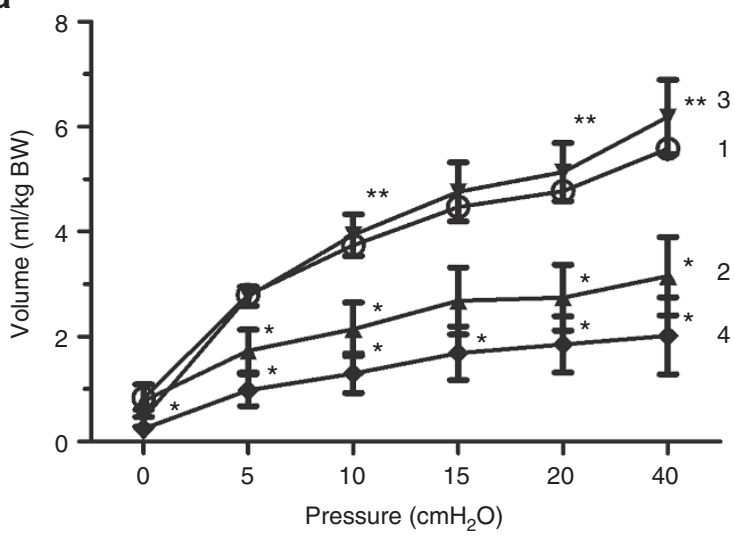

C

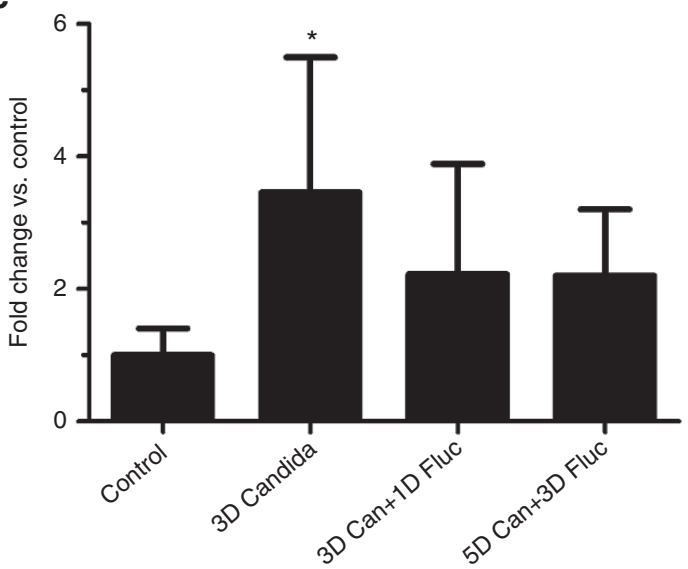

b

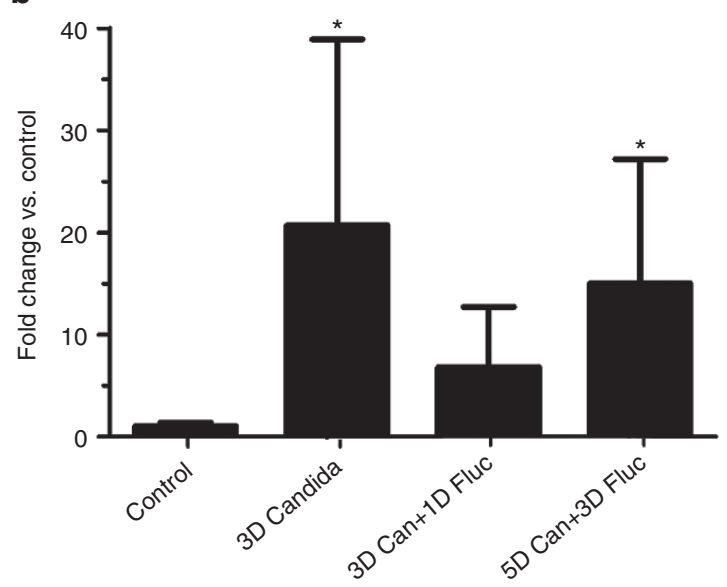

d

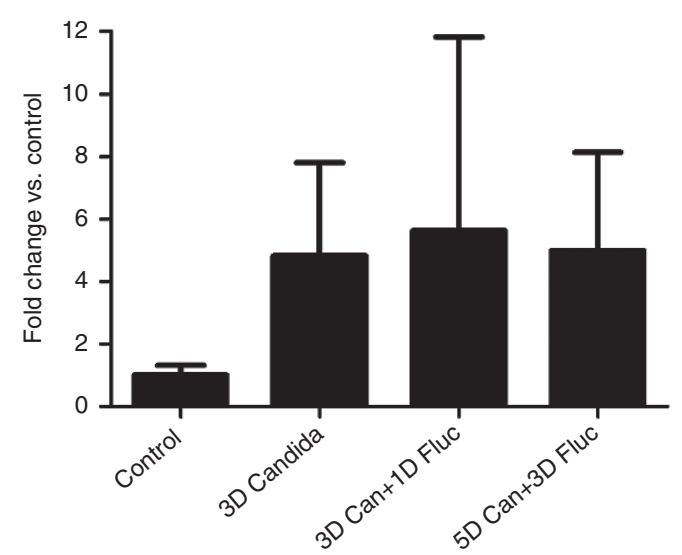

Figure 3. Lung gas volume and surfactant protein mRNA. (a) Pressure-volume curves demonstrated a significantly decreased lung volume in the $3 \mathrm{~d} C$. albicans group and the $5 \mathrm{~d}$ C. albicans plus $3 \mathrm{~d}$ fluconazole group relative to control. The lung gas volumes significantly increased in the $3 \mathrm{~d} C$. albicans plus $1 \mathrm{~d}$ fluconazole group at 10,20, and $40 \mathrm{cmH}_{2} \mathrm{O}$ relative to the $3 \mathrm{~d}$ C. albicans group. (1, control group; 2, $3 \mathrm{~d}$ C. albicans group; $3,3 \mathrm{~d}$ C. albicans plus $1 \mathrm{~d}$ fluconazole group; $4,5 \mathrm{~d}$ C. albicans plus $3 \mathrm{~d}$ fluconazole group). (b-d) Quantification of mRNAs for SP-A, SP-B, and SP-D, respectively, was performed using real-time PCR with ovine-specific primers. SP-A mRNA levels were significantly increased in the $3 \mathrm{~d}$ C. albicans group and $5 \mathrm{~d}$ C. albicans plus $3 \mathrm{~d}$ fluconazole group relative to control. SP-B levels were significantly increased in the $3 \mathrm{~d}$ C. albicans group relative to control $(* P<0.05$ vs. control, $* * P<0.05$ vs. the $3 \mathrm{~d}$ C. albicans group).

less systemic inflammation and inconsistent lung maturation (17). Intra-amniotic C. albicans causes a substantially different inflammatory response, characterized by severe inflammation in the fetal lung, inflammation and edema of the fetal skin (18), and fetal death beyond $3 \mathrm{~d}$ of exposure. Interestingly, despite a robust intrauterine response, there were no overt effects on the well-being of the ewe, further underscoring the potential for significant fetal involvement to adopt a subclinical course. There is less histological chorioamnionitis observed with $C$. albicans, but the multiorgan fetal effects are far more severe. At $3 \mathrm{~d}$ postfetal exposure, lung cytokine levels are very high, and inflammatory cells are activated in the lungs. However, there is lung maturation response characterized by increased SP-A, SP-B, and SP-D mRNA levels. There is no lung maturation as assessed by a pressure volume curve, probably due to the progressive pneumonia caused by $C$. albicans. Of the C. albicansexposed animals, 5 of 18 fetuses died, and $72 \%$ of the survivors had positive blood cultures at delivery. This demonstrates the ability of C. albicans to cause acute sepsis in these animals.
Intrauterine fluconazole treatment decreased the adverse effects of intra-amniotic C. albicans infection. mRNA expression of IL-6 MCP-1, CD3, and PU.1-positive cell counts in the fetal lung significantly decreased in the $3 \mathrm{~d}$ C. albicans plus 1 $\mathrm{d}$ fluconazole group relative to the $3 \mathrm{~d} C$. albicans group. In addition, the lung gas volume in the $3 \mathrm{~d} C$. albicans plus $1 \mathrm{~d}$ fluconazole group significantly increased compared with the $3 \mathrm{~d}$ C. albicans group. This is consistent with a reduction of lung inflammation in the $3 \mathrm{~d} C$. albicans plus $1 \mathrm{~d}$ fluconazole group. In the chorioamnion, mRNA expression of IL-1 $\beta$, IL-6, and MCP-1 significantly decreased in the $3 \mathrm{~d} C$. albicans plus $1 \mathrm{~d}$ fluconazole group relative to the $3 \mathrm{~d} C$. albicans group. Although the culture results demonstrated that most of the $C$. albicans-exposed animals had positive cultures, irrespective of the fluconazole treatment, the amount of Candida RNA in the fetal lung was decreased by $1 \mathrm{~d}$ of fluconazole $(P=0.06)$ (Table 1). Fluconazole is a fungal-static drug and would not be expected to clear the infection without effective innate immune responses, which may be minimal in these naive 
a

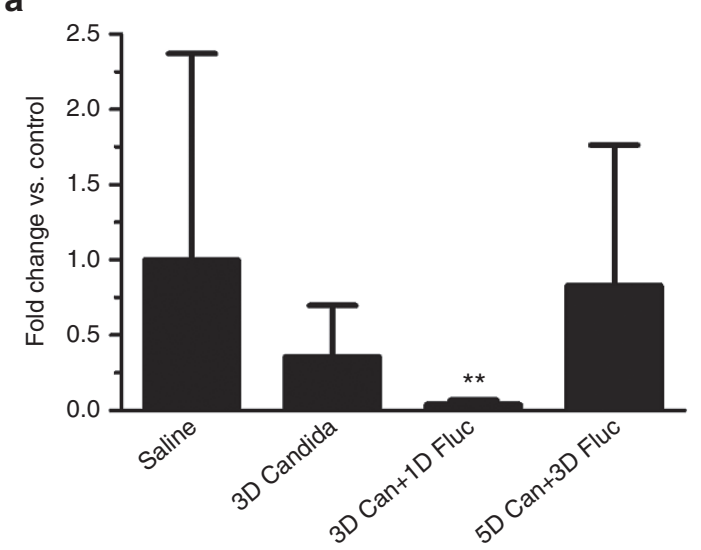

C

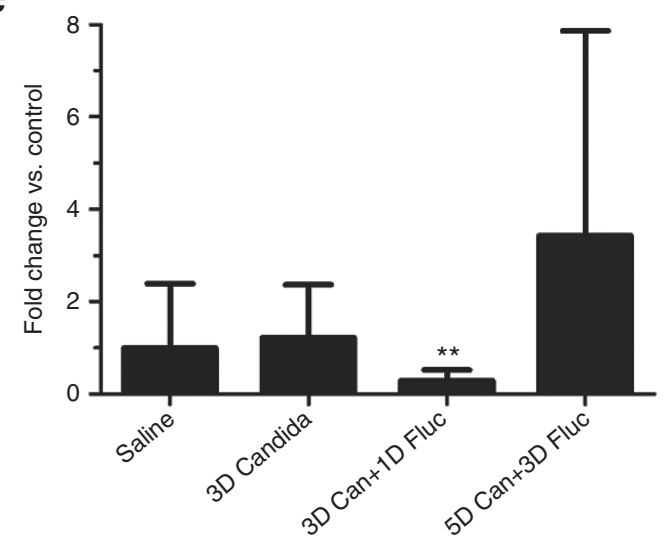

b
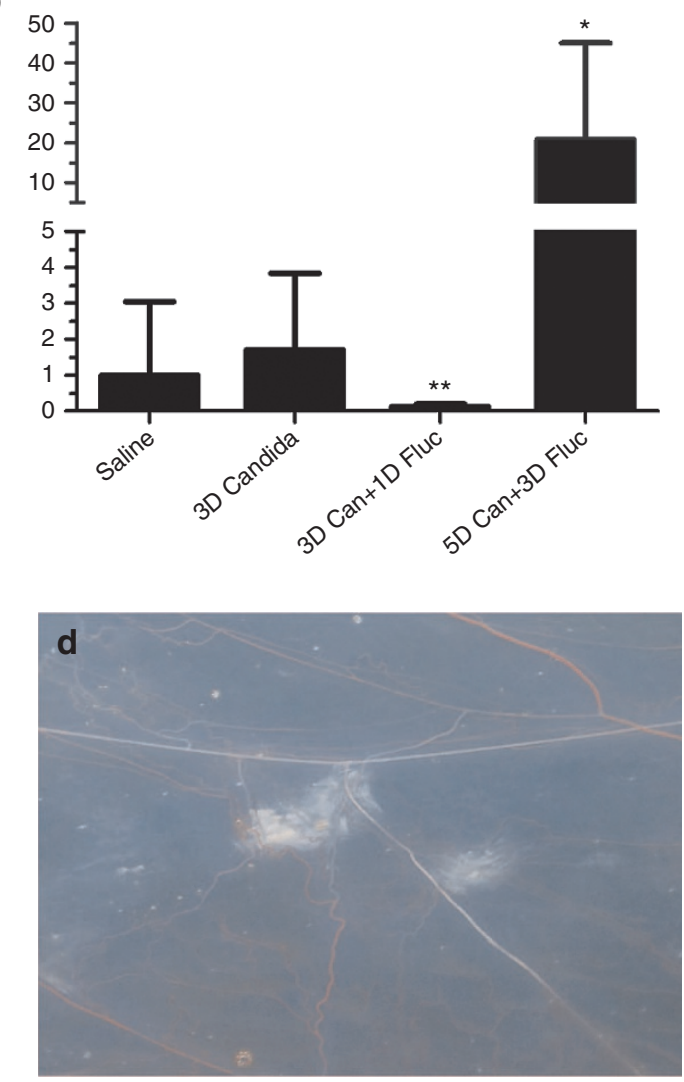

Figure 4. Cytokine expression in the chorioamnion following intra-amniotic C. albicans infection and fluconazole treatment. (a-c) Quantification of mRNA for IL-1 $\beta$, IL-6, and MCP-1 respectively was performed using real-time PCR with ovine-specific primers. Levels for each group were expressed as fold increase relative to control. Only mRNA expression of IL-6 significantly increased in the $5 \mathrm{~d}$ C. albicans plus $3 \mathrm{~d}$ fluconazole group relative to control. In the $3 \mathrm{~d}$ C. albicans plus $1 \mathrm{~d}$ fluconazole group, mRNA expression of IL-1 $\beta$, IL-6, and MCP-1 significantly decreased compared with the $3 \mathrm{~d} C$. albicans group. (d) Representative photograph shows large white plaques on the chorioamnion membrane ( ${ }^{*} P<0.05$ vs. control, ${ }^{*} P<0.05$ vs. the $3 \mathrm{~d} C$. albicans group).

premature fetuses. Nevertheless, a single intra-amniotic and fetal intraperitoneal dose resulted in survival for five of eight animals exposed to C. albicans for greater than $4 \mathrm{~d}$ despite a significantly increased inflammatory response relative to control animals. However: the increase in inflammatory markers in multiple organs; the presence of viable $C$. albicans in the amniotic fluid; a $\sim 7$-fold increase in mean C. albicans RNA in the fetal lung; increases in both CD3-positive cell counts and PU.1-immunostained inflammatory cells; decreases in lung volume as indicated by pressure-volume curves and increases in the weight of the posterior mediastinal lymph node; in 5 $\mathrm{d}$-treated compared to $3 \mathrm{~d}$-treated animals, all indicate continued infection. The initial benefit of fluconazole treatment in the 3-d group was lost $24-72 \mathrm{~h}$ post-treatment. Based on our previous studies, it is likely all fetuses would have died beyond $5 \mathrm{~d}$ as a result of a re-established C. albicans infection (18).

Although there have been many previous attempts to reduce the rate of preterm birth with the use of antibiotic therapy, all with varying results (28), very few studies have demonstrated the effects of intrauterine antifungal therapy in pregnant women with Candida spp. infection. In one report, a pregnant woman with premature rupture of membranes and intra-amniotic infection with $C$. albicans was treated with transcervical amniofusion of amphotericin B and the infant was delivered without complications (29). Recently, Bean et al. (19) reported the resolution of two cases of intra-amniotic $C$. albicans infection with maternal and intra-amniotic fluconazole treatment. Thus, treatment of intra-amniotic C. albicans infection with intrauterine antifungal therapy may be helpful but further studies are needed to evaluate the effectiveness and possible toxicity of the therapeutic agent.

A limitation of this study is the absence of measurements of fluconazole levels within the fetal circulation, making it difficult to determine whether the five fetal deaths that occurred among the 16 animals comprising the fluconazole-treated groups are due, at least in part, to fetotoxic drug effects. The use of a combined intra-amniotic and fetal intraperitoneal dosing strategy was designed to ensure drug delivery to both the fetus and amniotic fluid. A drawback to this approach is that we are unable to determine differences or additive effects in therapeutic efficacy or fetotoxicity conveyed by these two delivery strategies. There are concerns relating to the use of fluconazole in pregnancy; it has been shown to have teratogenic effects in several animal model studies and recent in vivo work in a mouse model suggests that fluconazole perturbs CYP 26, an enzyme involved in retinoic acid homeostasis (30). 
a

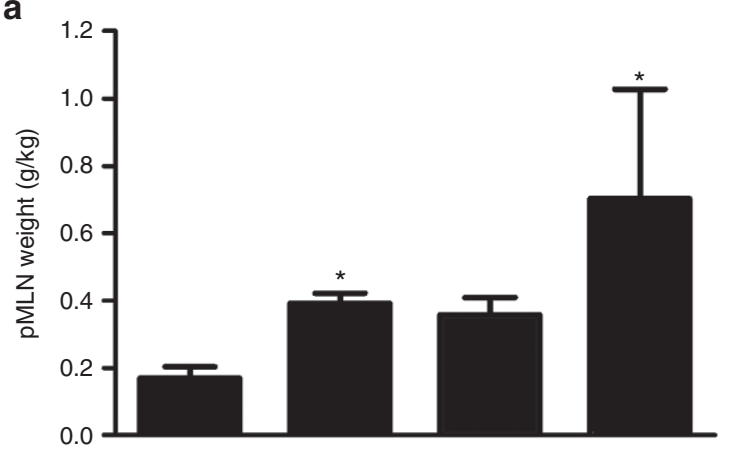

C

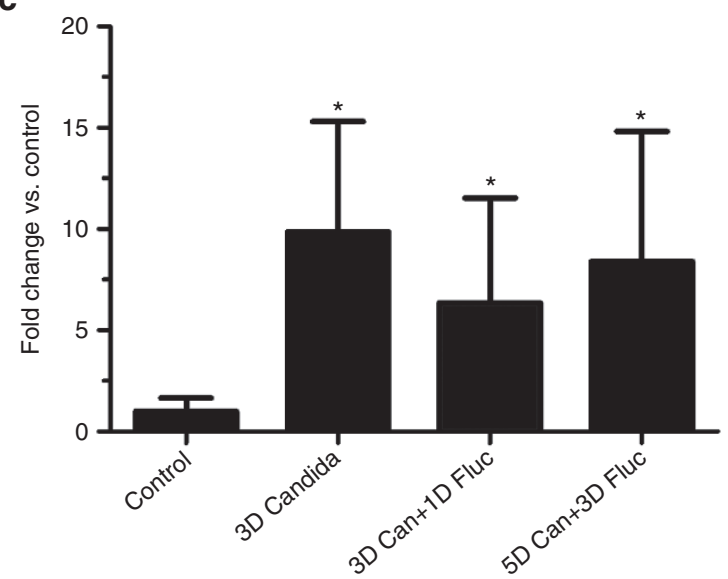

b

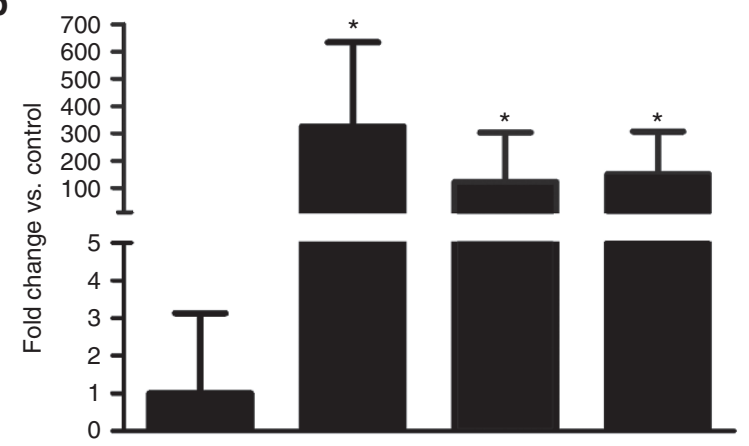

d

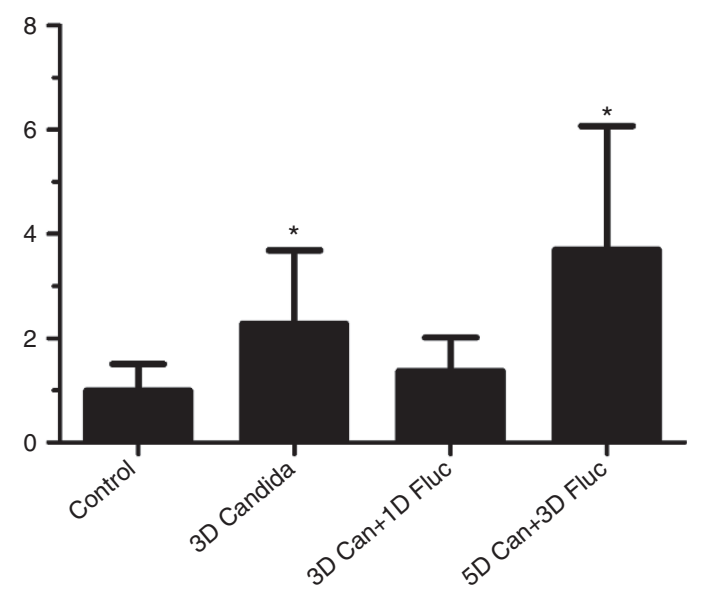

Figure 5. Systemic responses to C. albicans. (a) The weight of the posterior mediastinal lymph node significantly increased in the $3 \mathrm{~d}$ C. albicans group and $5 \mathrm{~d}$ C. albicans plus $3 \mathrm{~d}$ fluconazole group relative to control. $(\mathbf{b}, \mathbf{c})$ Acute phase protein gene expression in the liver. Quantification of mRNAs for SAA3 and C-reactive protein (CRP), respectively, was performed using real-time PCR with ovine-specific primers and probes. SAA3 and CRP significantly increased in all groups relative to control. (d) Quantification of mRNA for IL- 6 in the spleen significantly increased in the $3 \mathrm{~d} C$. albicans group and $5 \mathrm{~d} C$. albicans plus $3 \mathrm{~d}$ fluconazole group relative to control $(* P<0.05$ vs. control).

Interestingly, fluconazole treatment was well tolerated in the control group, suggesting that if fluconazole does have fetotoxic effects it may be more pronounced in conjunction with a pre-existing pathology or fetal stress, including intrauterine infection. Perhaps reassuringly, a number of retrospective cohort studies have failed to demonstrate an increased risk of congenital malformation in association with fluconazole use in pregnancy $(22,31,32)$. Irrespective, further studies are warranted in order to determine the potential adverse effects deriving from exposing the fetus to high levels of fluconazole. A further limitation of this study was our decision to deliver two of the animals in the $5 \mathrm{~d}$ C. albicans plus $3 \mathrm{~d}$ fluconazole group 24 h early (equivalent to $4 \mathrm{~d}$ C. albicans and $2 \mathrm{~d}$ fluconazole exposure) due to concerns over fetal well-being. Although we are able to demonstrate that treatment efficacy was similarly reduced in these animals, data from this group does need to be interpreted with this difference in exposure length in mind.

In the current study, we administered a high dose by intraamniotic and fetal intraperitoneal injections but failed to clear C. albicans from the amniotic fluid within $24 \mathrm{~h}$. The efficacy of drugs may be altered in pregnant women due to physiologic changes and some drugs require higher dosing as a result of changes in fluid volumes. Administration of medication by amniocentesis facilitates the direct treatment of the fetus (19).
Fluconazole is absorbed orally and thus could achieve continuous but decreasing fetal exposures with fetal swallowing. Our novel data demonstrate that although intra-amniotic fluconazole therapy for intrauterine C. albicans infection initially decreases adverse fetal effects, as indicated by a reduction in the lung and chorioamnion inflammation in a single-dose regimen, it is unlikely to prolong fetal survival beyond $5 \mathrm{~d}$. A better understanding of the host immune response to C. albicans infection and as well as responses to multiple dose antifungal treatment regimens will help in the design of new therapies in perinatal medicine.

\section{METHODS}

\section{Animals}

All procedures involving animals were conducted at The University of Western Australia (Perth, WA) following review and approval by the animal care and use committees of The University of Western Australia and Cincinnati Children's Hospital (Cincinnati, OH). Datemated Merino ewes with singleton pregnancies were randomized to one of the following exposure groups: (i) Control: intra-amniotic injection of $2 \mathrm{ml}$ saline $(n=11)$ or intra-amniotic and fetal intraperitoneal injections of fluconazole (Claris Lifesciences Limited, Ahmedabad, India) $(n=5)$; or (ii) C. albicans at day 3 prior to delivery: intra-amniotic injection of $10^{7}$ colony-forming unit (CFU) C. albicans in $2 \mathrm{ml}$ saline $3 \mathrm{~d}$ prior to delivery $(n=8$; one animal was found to be uninfected at postmortem and was eliminated from subsequent analyses, yielding a group $n$ of 7); or (iii) C. albicans at day 3 prior 
to delivery and fluconazole at day 1 prior to delivery: intra-amniotic injection of $10^{7} \mathrm{CFU}$ C. albicans in $2 \mathrm{ml}$ saline $3 \mathrm{~d}$ prior to delivery, followed by intra-amniotic and fetal intraperitoneal injections of fluconazole $1 \mathrm{~d}$ prior to delivery, yielding $3 \mathrm{~d}$ of antenatal C. albicans exposure and $1 \mathrm{~d}$ of antenatal fluconazole exposure $(n=8)$; or (iv) C. albicans at day 5 prior to delivery and fluconazole at day 3 prior to delivery: intra-amniotic injection of $10^{7} \mathrm{CFU}$ C. albicans $5 \mathrm{~d}$ prior to delivery followed by intra-amniotic and fetal intraperitoneal injections of fluconazole $3 \mathrm{~d}$ prior to delivery, yielding $5 \mathrm{~d}$ of antenatal $C$. albicans exposure and $3 \mathrm{~d}$ of antenatal fluconazole exposure $(n=8)$.

The ultrasound guided dose of fluconazole was $30 \mathrm{mg}$ per injection $(12 \mathrm{mg} / \mathrm{kg}$ based on an estimated fetal weight of $2.5 \mathrm{~kg})$ and was divided and given in equal $15 \mathrm{mg}$ doses by intra-amniotic and fetal intraperitoneal injections of $7.5 \mathrm{ml}$ each. Fluconazole was selected on the expectation that its prior successful use in treating intra-amniotic candidiasis, long half-life and the potential for uptake from AF swallowed by the fetal gut would allow us to deliver a lasting therapeutic dose from a single intra-amniotic and fetal intraperitoneal administration $(20,21)$. Successful placement of intra-amniotic injections were confirmed with electrolyte (Cl-) analysis of amniotic fluid using a Siemens Rapidlab 1265 Analyser (Siemens, Munich, Germany). Successful placement of fetal intraperitoneal injections were confirmed by ultrasound visualization of fluid after injection. Combined intra-amniotic and fetal intraperitoneal injections were administered in an attempt to promote high fetal uptake of fluconazole as data on the bioavailability of this drug within sheep are not known.

All fetuses were surgically delivered at $122 \pm 1$ day GA, and euthanized with intravenous pentobarbital $(100 \mathrm{mg} / \mathrm{kg})$. Each fetus was weighed and fetal cord blood was collected for plasma, cell counts (VetPath, Perth, Western Australia), blood gas, and $\mathrm{pH}$ measurements. Fetal lung fluid and tissues for protein and mRNA expression analyses were collected at autopsy and snap frozen in liquid nitrogen. For determination of pulmonary compliance, a deflation air pressure-volume curve was measured from a static inflation of $40 \mathrm{cmH}_{2} 0$ airway pressure with the chest open (33).

\section{C. albicans Culture and Detection}

A Western Australian clinical isolate of C. albicans (19) was cultured on Difco Sabaraud-Dextrose agar (Becton Dickinson, Franklin Lakes, $\mathrm{NJ})$ at $37^{\circ} \mathrm{C}$ for $48 \mathrm{~h}$ and single colonies were inoculated into sterile phosphate-buffered saline (PBS) (Sigma-Aldrich, St. Loius, MO) (18). C. albicans colonial morphology was confirmed by growth on Brilliance Candida Agar (Oxoid, Adelaide, Australia). Inoculums were quantified using a plate dilution series as per standard microbiological methods and recorded as $\mathrm{CFU} / \mathrm{ml}$. Quantified inoculums $\left(10^{7}\right.$ $\mathrm{CFU}$ in $2 \mathrm{ml} \mathrm{PBS}$ ) were stored at $-80{ }^{\circ} \mathrm{C}$ prior to use. For amniotic fluid culture, $100 \mu \mathrm{l}$ of fresh amniotic fluid was inoculated onto Difco Sabaraud-Dextrose agar and evenly distributed across the plate with a sterile spreader. Incubation conditions were as described above. For blood culture, $2 \mathrm{ml}$ fetal blood samples were inoculated into BACTEC Peds Plus culture vials (Becton Dickinson, Franklin Lakes, NJ) and incubated aerobically at $37^{\circ} \mathrm{C}$ for $72 \mathrm{~h}$. Every $24 \mathrm{~h}$, a $1 \mathrm{ml}$ sample was aseptically removed and $100 \mu \mathrm{l}$ of this was subcultured on $5 \%$ sheep blood agar (Pathwest Laboratories, Perth, Western Australia) at $37^{\circ} \mathrm{C}$ for $48 \mathrm{~h}$. For both amniotic fluid and blood cultures, C. albicans colonial morphology was confirmed as described above. RNA extracted from fetal lung was screened using a real-time PCR assay targeting the RNase P RNA gene of C. albicans (19).

\section{Quantification of mRNA Expression}

Total RNA was extracted from fetal lung, chorioamnion, liver, and spleen tissues and homogenized in TRIzol (Life Technologies, Carlsbad, CA) as per manufacturer's instructions, and mRNA quantitation was performed by real-time PCR (27). mRNA was reverse transcribed to yield double-stranded cDNA (Verso cDNA kit, Thermo Scientific, Waltham, MA), which was used as template in real-time PCR assays with ovinespecific primers/Taqman probes (Applied Biosystems, Carlsbad CA) (34). Gene expression was measured for cytokines: IL- $1 \alpha$, IL-1 $\beta$, IL-6, IL-8, TNF- $\alpha$, monocyte chemotactant protein-1 (MCP-1), serum amyloid protein A3 (SAA3), and CRP. The values for each mRNA target were normalized to internal $18 \mathrm{~S}$ rRNA values. Final expression data are represented as fold increase relative to control values.

\section{Histology}

Five-micrometer-thick sections from formalin-fixed lung and chorioamnion tissues embedded in paraffin blocks were stained with hematoxylin and eosin. Sections for immunohistochemical analysis were deparaffinized and rehydrated before microwave-assisted antigen retrieval in citric acid buffer at $\mathrm{pH}$ 6.0. Endogenous peroxidase activity was blocked with $\mathrm{CH}_{3} \mathrm{OH} / \mathrm{H}_{2} \mathrm{O}_{2}$. Sections were blocked with $2 \%$ goat serum in PBS. Sections were incubated for $16 \mathrm{~h}$ at $4{ }^{\circ} \mathrm{C}$ with primary antibodies specific for either PU.1 (Sc-352, Santa Cruz Biotechnology, CA, 1:400 dilution), CD3 (A0452, Dako, Glostrup, Denmark, 1:50) or MPO (catalogue \#CMC028 Cell Marque, Rocklin, CA, 1:50) diluted in $2 \%$ goat serum in PBS. Sections were washed repeatedly in PBS before being incubated with an appropriate species-specific secondary antibody (1:200) for $30 \mathrm{~min}$ at room temperature. Slides were repeatedly washed in PBS before antigen:antibody complexes were visualized with a Vectastain $A B C$ peroxidase Elite kit (Vector Laboratories, Burlingame, CA). Antigen detection was enhanced with nickel-diaminobenzidine, followed by incubation with TRIS-cobalt to give a black precipitate. Nuclei were counterstained with Nuclear Fast Red for photo-microscopy. Blind scoring of tissues was done by counting PU.1-, CD-3-, or MPO-positive cells in 10 comparable nonoverlapping high power fields ( $\times 40$ objective).

\section{Statistical Analyses}

All values are expressed as mean \pm SD. Initial comparisons were made using Kruskal-Wallis one-way ANOVA across the four study groups. Selective comparisons between groups were made with two-tailed unpaired $t$-tests. Nonparametric data were tested for significance with Mann-Whitney tests. Statistical analysis was performed by GraphPad Prism v5.0. Significance was accepted at the level of $P<0.05$.

\section{ACKNOWLEDGMENTS}

The authors wish to gratefully acknowledge the support of Siemens Australia for donating the Rapidlab 1200 reagents used in this study.

\section{STATEMENT OF FINANCIAL SUPPORT}

This work was supported by National Institutes of Health (Bethesda, MD) grant (HD 57869) to S.G.K. and by the Financial Markets Foundation for Children (Sydney, Australia) grant (EOI-2013-059) to M.W.K. M.W.K. is supported by the Women and Infants Research Foundation (Perth, Western Australia).

\section{Disclosure: None declared.}

\section{REFERENCES}

1. Goldenberg RL, Culhane JF, Iams JD, Romero R. Epidemiology and causes of preterm birth. Lancet 2008;371:75-84.

2. DiGiulio DB. Diversity of microbes in amniotic fluid. Semin Fetal Neonatal Med 2012;17:2-11.

3. Goldenberg RL, Hauth JC, Andrews WW. Intrauterine infection and preterm delivery. N Engl J Med 2000;342:1500-7.

4. Jones HE, Harris KA, Azizia M, et al. Differing prevalence and diversity of bacterial species in fetal membranes from very preterm and term labor. PLoS One 2009; 4:e8205.

5. Hay P, Czeizel AE. Asymptomatic trichomonas and candida colonization and pregnancy outcome. Best Pract Res Clin Obstet Gynaecol 2007;21:403-9.

6. Chaim W, Mazor M, Wiznitzer A. The prevalence and clinical significance of intraamniotic infection with Candida species in women with preterm labor. Arch Gynecol Obstet 1992;251:9-15.

7. DiGiulio DB, Romero R, Amogan HP, et al. Microbial prevalence, diversity and abundance in amniotic fluid during preterm labor: a molecular and culture-based investigation. PLoS One 2008;3:e3056.

8. Combs CA, Gravett M, Garite TJ, et al.; ProteoGenix/Obstetrix Collaborative Research Network. Amniotic fluid infection, inflammation, and colonization in preterm labor with intact membranes. Am J Obstet Gynecol 2014;210:125.e1-125.e15. 
9. Marelli G, Mariani A, Frigerio L, Leone E, Ferrari A. Fetal Candida infection associated with an intrauterine contraceptive device. Eur J Obstet Gynecol Reprod Biol 1996;68:209-12.

10. Benjamin DK Jr, Stoll BJ, Fanaroff AA, et al.; National Institute of Child Health and Human Development Neonatal Research Network. Neonatal candidiasis among extremely low birth weight infants: risk factors, mortality rates, and neurodevelopmental outcomes at 18 to 22 months. Pediatrics 2006;117:84-92.

11. Darmstadt GL, Dinulos JG, Miller Z. Congenital cutaneous candidiasis: clinical presentation, pathogenesis, and management guidelines. Pediatrics 2000;105:438-44.

12. Cotch MF, Hillier SL, Gibbs RS, Eschenbach DA. Epidemiology and outcomes associated with moderate to heavy Candida colonization during pregnancy. Vaginal Infections and Prematurity Study Group. Am J Obstet Gynecol 1998;178:374-80.

13. Czeizel AE, Fladung B, Vargha P. Preterm birth reduction after clotrimazole treatment during pregnancy. Eur J Obstet Gynecol Reprod Biol 2004;116:157-63.

14. Roberts CL, Rickard K, Kotsiou G, Morris JM. Treatment of asymptomatic vaginal candidiasis in pregnancy to prevent preterm birth: an openlabel pilot randomized controlled trial. BMC Pregnancy Childbirth 2011;11:18.

15. Snyder CC, Wolfe KB, Gisslen T, et al. Modulation of lipopolysaccharideinduced chorioamnionitis by Ureaplasma parvum in sheep. Am J Obstet Gynecol 2013;208:399.e1-8.

16. Kallapur SG, Nitsos I, Moss TJ, et al. IL-1 mediates pulmonary and systemic inflammatory responses to chorioamnionitis induced by lipopolysaccharide. Am J Respir Crit Care Med 2009;179:955-61.

17. Moss TJ, Knox CL, Kallapur SG, et al. Experimental amniotic fluid infection in sheep: effects of Ureaplasma parvum serovars 3 and 6 on preterm or term fetal sheep. Am J Obstet Gynecol 2008;198:122.e1-8.

18. Payne MS, Kemp MW, Kallapur SG, et al. Intrauterine Candida albicans infection elicits severe inflammation in fetal sheep. Pediatr Res 2014;75:716-22.

19. Bean LM, Jackson JR, Dobak WJ, Beiswenger TR, Thorp JA. Intra-amniotic fluconazole therapy for Candida albicans intra-amniotic infection. Obstet Gynecol 2013;121(2 Pt 2 Suppl 1):452-4.

20. Bean LM, Jackson JR, Dobak WJ, Beiswenger TR, Thorp JA. Intra-amniotic fluconazole therapy for Candida albicans intra-amniotic infection. Obstet Gynecol 2013;121(2 Pt 2 Suppl 1):452-4.
21. Brammer KW, Coates PE. Pharmacokinetics of fluconazole in pediatric patients. Eur J Clin Microbiol Infect Dis 1994;13:325-9.

22. Nørgaard M, Pedersen L, Gislum M, et al. Maternal use of fluconazole and risk of congenital malformations: a Danish population-based cohort study. J Antimicrob Chemother 2008;62:172-6.

23. Hedenstierna G, Lattuada M. Lymphatics and lymph in acute lung injury. Curr Opin Crit Care 2008;14:31-6.

24. El-Masry FA, Neal TJ, Subhedar NV. Risk factors for invasive fungal infection in neonates. Acta Paediatr 2002;91:198-202.

25. Oh KJ, Lee KA, Sohn YK, et al. Intraamniotic infection with genital mycoplasmas exhibits a more intense inflammatory response than intraamniotic infection with other microorganisms in patients with preterm premature rupture of membranes. Am J Obstet Gynecol 2010;203:211.e1-8.

26. DiGiulio DB, Romero R, Kusanovic JP, et al. Prevalence and diversity of microbes in the amniotic fluid, the fetal inflammatory response, and pregnancy outcome in women with preterm pre-labor rupture of membranes. Am J Reprod Immunol 2010;64:38-57.

27. Kemp MW, Kannan PS, Saito M, et al. Selective exposure of the fetal lung and skin/amnion (but not gastro-intestinal tract) to LPS elicits acute systemic inflammation in fetal sheep. PLoS One 2013;8:e63355.

28. Brocklehurst P, Gordon A, Heatley E, Milan SJ. Antibiotics for treating bacterial vaginosis in pregnancy. Cochrane Database Syst Rev 2013;1:CD000262.

29. Shalev E, Battino S, Romano S, Blondhaim O, Ben-Ami M. Intraamniotic infection with Candida albicans successfully treated with transcervical amnioinfusion of amphotericin. Am J Obstet Gynecol 1994;170(5 Pt 1):1271-2.

30. Tiboni GM, Marotta F, Carletti E. Fluconazole alters CYP26 gene expression in mouse embryos. Reprod Toxicol 2009;27:199-202.

31. Jick SS. Pregnancy outcomes after maternal exposure to fluconazole. Pharmacotherapy 1999;19:221-2.

32. Sorensen HT, Nielsen GL, Olesen C, et al. Risk of malformations and other outcomes in children exposed to fluconazole in utero. Br J Clin Pharmacol 1999;48:234-8.

33. Jobe AH, Newnham JP, Willet KE, et al. Endotoxin-induced lung maturation in preterm lambs is not mediated by cortisol. Am J Respir Crit Care Med 2000;162:1656-61.

34. Kallapur SG, Kramer BW, Nitsos I, et al. Pulmonary and systemic inflammatory responses to intra-amniotic IL-1 $\alpha$ in fetal sheep. Am J Physiol Lung Cell Mol Physiol 2011;301:L285-95. 